\title{
The Effect of Vacuum Pressure During Fabrication of Zinc Sulphide Thin Film Using Thermal Evaporator
}

\author{
Bassam Khaled Balkhair Almashgari and Saifful Kamaluddin Muzakir* \\ Material Technology Programme, Faculty of Industrial Sciences and Technology, Universiti Malaysia Pahang, Lebuhraya Tun Razak, Gambang, \\ 26300 Kuantan, Pahang, Malaysia
}

\begin{abstract}
Zinc sulphide is a remarkable semiconductor material, with its highly demanded optical, electrical properties and its availability, it is used in the field of photovoltaic applications. The objectives are to investigate the effect of vacuum pressure on the glass substrate and the study different cluster models based on its crystal size. The ZnS thin films were fabricated using a thermal evaporator at the pressure of $10^{-5}$ Torr, $10^{-6}$ Torr, and $10^{-7}$ Torr. Four different size cluster models have been characterized theoretically. Each model was built based on the size of crystal structures, optimized to the lowest energy level, and evaluated as realistic clusters. The structural and optical properties of the thin films were studied using X-ray diffractometry, absorption, and photoluminescence spectroscopy respectively. Significant differences were observed in the crystal structure and optical properties of fabricated $\mathrm{MoS}_{2}$ thin films at different pressures.
\end{abstract}

\section{ARTICLE HISTORY}

Received: $6^{\text {th }}$ Aug 2020

Revised: $10^{\text {th }}$ Dec 2020

Accepted: 25 $5^{\text {th }}$ Feb 2021

\section{KEYWORDS}

ZnS, Vacuum Thermal

Evaporation, Density

Functional Theory

\section{INTRODUCTION}

Zinc sulphide has been used in diverse applications, such as LED, flat panel displays, infrared windows, and sensors. There are similarities between $\mathrm{ZnO}$ and $\mathrm{ZnS}$ regarding their atomic structure and chemical properties. The $\mathrm{ZnS}$ and $\mathrm{ZnO}$ have a large band gap ca. $3.77 \mathrm{eV}$ and $3.4 \mathrm{eV}$ [1], which has to lead their applications in electroluminescence devices and visible-blind ultraviolet (UV) photodetectors respectively. Solar cells are in the focus of researchers due to their ability to convert the energy of light into electrical energy. The free, and renewable fuel (light) would offer zero harmful byproducts during the photovoltaic (PV) conversion mechanism. The commercial electrical energy generation however is still highly dependable to non-renewable energy resources i.e., coal, peat, and oil, which requires burning of the fuels that would cause an increment of $\mathrm{CO}_{2}$ emissions to the environment [2].

There are three classifications of solar cells based on the physical state of the photo-active material used. The first category of solar cell is based on bulk silicon which has recorded the highest photovoltaic conversion efficiency (PCE) for a single cell without a concentrator ca. $26.1 \%$. The second category is the thin-film solar cell which has recorded PCE ca. 23.4\% with copper-indium-gallium-selenide (CIGS) device structure without concentrator. Other device structures for this category of solar cell e.g., CdTe (PCE ca. 22.1\%), and hydrogenated amorphous silicon solar cell (PCE ca. 14.0\%). The third category is the emerging PV which highlighted the perovskite solar cell that has recorded PCE as high as $25.5 \%$. Other device structures in this category of solar cell e.g., dye-sensitized solar cell (DSSC) with PCE ca. 13.0\%, organic cell with PCE ca. 18.2\%, inorganic cell (CZTSSe) with PCE ca. 12.6\% and quantum dots solar cell (QDSC) with PCE ca. $18.1 \%$.

\section{MATERIALS AND METHOD}

\section{Synthesis of $\mathrm{ZnS}$ thin film}

Zinc powder (Merck), sulphur powder (Merck), fluorine-doped tin oxide (FTO) conducting glass (Solaronix), thermal evaporator (TE MSSLAB/200), Field Emission Scanning Electron Microscope (JEOL JSM 7800F FESEM), Rigaku Miniflex II X-ray Diffractometer (XRD), absorption spectrometer (Shimadzu UV-2600) and photoluminescence spectrometer (NIR 300/2 Edinburgh Instruments) were used throughout the work. Zinc powder $0.065 \mathrm{~g}$ mixed with 0.032 $\mathrm{g}$ of sulphur $(\mathrm{S})$ and loaded in molybdenum boat and placed in the vacuum chamber of the thermal evaporator. A conducting glass was placed $5 \mathrm{~cm}$ above the boat on the stage for sample. The vacuum level of the chamber was set to three different pressures i.e., $10^{-5}$ Torr, $10^{-6}$ Torr, and $10^{-7}$. The reactants were thermally evaporated at the voltage and current of $1.63 \mathrm{~V}$ and $65 \mathrm{~A}$ respectively and deposited on the FTO glass. The fabricated thin films of ZnS later cooled down at room temperature.

The crystal structure of the thin films fabricated at different pressures was investigated using Rigaku Miniflex II Xray Diffractometer, with a wavelength of $1.54 \AA$ generated at $30 \mathrm{kV}$ and $15 \mathrm{~mA}$ with a step width $0.01^{\circ}$ in the range $10^{\circ}$ to $80^{\circ}$. Morphological study of the thin films was conducted using JEOL JSM 7800F FESEM. Absorption spectrometer (Shimadzu UV-2600) was used to study the optoelectronic properties of the films. The excitonic transition peaks were 
determined using graph fitting to four Gaussian peaks which correspond to electronic transitions of the electron from ground to excited states. Edinburgh Instruments FS920 photoluminescence spectrometer was used to study the characteristic of radiative electron-hole recombinations.

\section{Realistic clusters}

$\mathrm{ZnS}$ nanoclusters were modeled based on their bulk crystallographic profile. The built models were optimized to the lowest energy structure using density functional theory (DFT) calculations at b3lyp/lanl2dz level, followed by harmonic frequency calculations. Models with negative frequencies were discarded at this stage which corresponds to non-realistic models. The optoelectronic properties (i.e., ground state, excited state, and bandgap) of the models were determined using time-dependent density functional theory (TD-DFT) calculations.

\section{EXPERIMENTAL RESULTS}

\section{Crystal structure}

The analyzed XRD data of $\mathrm{ZnS}$ thin film in the range of $10^{\circ}$ to $80^{\circ}$ shows hexagonal (wurtzite) structure (Figure 1) [3]. The reflecting planes of [002], [111], [200] and [110] were observed at $23.91^{\circ}, 28.63^{\circ}, 32.19^{\circ}$, and $43.61^{\circ}$ of diffraction angles respectively in $\mathrm{ZnS}$ fabricated under vacuum pressure of $10^{-5}$ Torr. A significant shift of position of diffraction angles which correspond to [002], [101], [111], [200], and [102] crystal plane could be observed at 23.91 ${ }^{\circ}$, $26.65^{\circ}, 28.63^{\circ}, 32.19^{\circ}$, and $38.66^{\circ}$ respectively for $\mathrm{ZnS}$ fabricated under the pressure of $10^{-6}$ Torr. For $\mathrm{ZnS}$ thin film fabricated under vacuum pressure of $10^{-7}$ Torr, the diffraction angles of [002], [111], [200] and [110] crystal planes could be observed at $23.91^{\circ}, 28.63^{\circ}, 32.19^{\circ}$ and $43.61^{\circ}$ respectively.

(a)
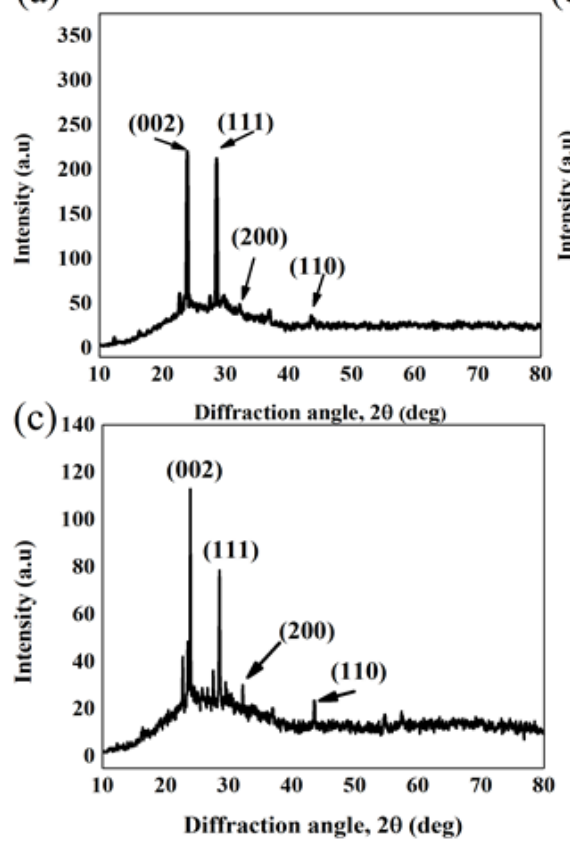

(b)

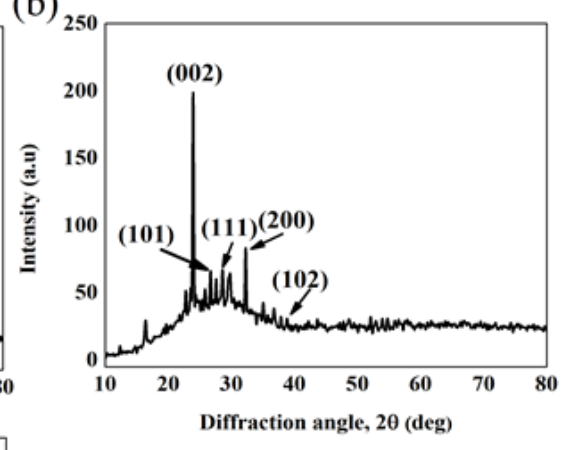

Figure 1: XRD patterns of ZnS thin films fabricated at (a) $10^{-5}$ Torr, (b) $10^{-6}$ Torr, (c) $10^{-7}$ Torr.

\section{Realistic ZnS Cluster Modelling}

Four clusters of $(\mathrm{ZnS})_{\mathrm{n}}$ (which $\mathrm{n}=3,6,9$, and 12) were modeled based on bulk crystallographic profile and optimized to the lowest energy structure as indicated in Figure 2. B3LYP functional lanl2dz basis set was used throughout the calculations. The harmonic frequencies calculations show that the structures are realistic due to all the frequencies are positive. The optoelectronic properties of each cluster such as band gap, ground state energy level (the highest occupied molecular orbital), and excited-state energy level (the lowest unoccupied molecular orbital) were calculated using TDDFT and presented in Table 1. It is observed that the bandgap of the $\mathrm{ZnS}$ is a function of the size of the $\mathrm{ZnS}$ cluster which is speculated due to the quantum confinement effect [4]. 
(a)

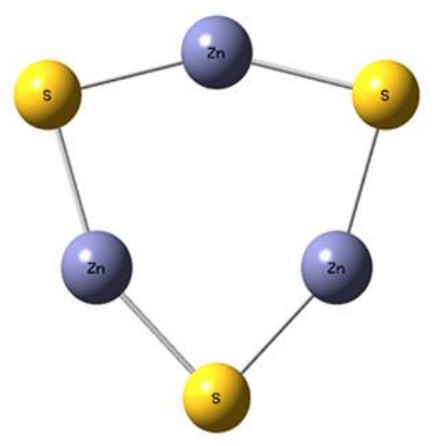

(c)

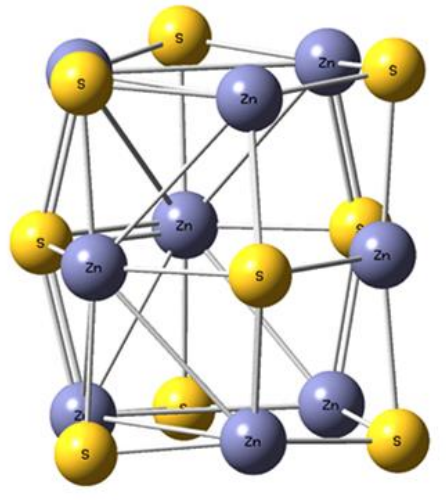

(b)

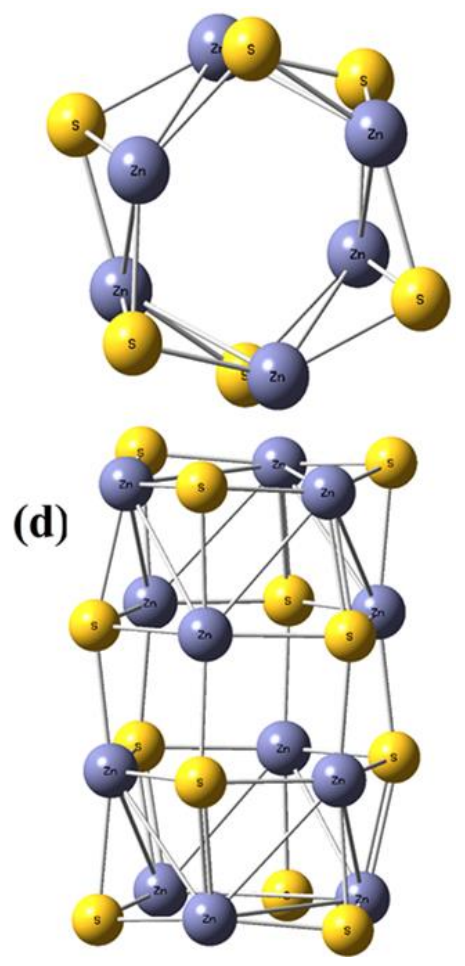

Figure 2: Optimized geometries of $(\mathrm{a})(\mathrm{ZnS})_{3}$, (b) $(\mathrm{ZnS})_{6},(\mathrm{c})(\mathrm{ZnS})_{9}$ and $(\mathrm{d})(\mathrm{ZnS})_{12}$.

Table 1: HOMO, LUMO, and bandgap values for each ZnS cluster.

\begin{tabular}{llll}
\hline Clusters & HOMO $(\mathbf{e V})$ & LUMO $(\mathbf{e V})$ & Band gap $(\mathbf{e V})$ \\
\hline$(\mathrm{ZnS})_{3}$ & -6.9416 & -3.2490 & 3.6926 \\
$(\mathrm{ZnS})_{6}$ & -7.1267 & -3.4232 & 3.7035 \\
$(\mathrm{ZnS})_{9}$ & -6.8927 & -3.4204 & 3.4723 \\
$(\mathrm{ZnS})_{12}$ & -6.8736 & -3.4559 & 3.4177 \\
\hline
\end{tabular}

\section{Optical Properties Analysis}

The optical properties of ZnS were analyzed by using an absorption spectrometer (Shimadzu UV-2600), after fitting the spectra to four Gaussian peaks as first, second, third, and fourth excitonic peaks as indicated in Figure 3. Focusing on the first excitonic peaks of the thin films and visualized model of $\mathrm{ZnS}$ cluster, a good agreement between $\mathrm{ZnS}$ thin film fabricated at a vacuum pressure of $10^{-7}$ Torr with $(\mathrm{ZnS})_{3}$ and $(\mathrm{ZnS})_{6}$ could be observed. From Tauc's plot in Figure 4, it is clearly shown that the bandgap increases with the increment of vacuum pressure, which corresponds to the decrement of the size of $\mathrm{ZnS}$ particles that act as basic building blocks of the thin films. The photoluminescence emission spectrum of $\mathrm{ZnS}$ thin films is plotted Figure 5. The plot provides four emission peaks at $413.633 \mathrm{~nm}, 469.153 \mathrm{~nm}$, 491.602 , and $714.313 \mathrm{~nm}$ for $\mathrm{ZnS}$ thin film fabricated at $10^{-5}$ Torr. $\mathrm{ZnS}$ thin film fabricated at $10^{-6}$ Torr shows emission peaks at 403.181 $\mathrm{nm}, 425.255 \mathrm{~nm}, 472.185 \mathrm{~nm}$, and $563.166 \mathrm{~nm}$. ZnS thin film fabricated at $10^{-7}$ Torr shows peaks at $421.585 \mathrm{~nm}, 470.523$ $\mathrm{nm}, 495.332 \mathrm{~nm}$, and $597.182 \mathrm{~nm}$. ZnS thin film fabricated at $10^{-7}$ have the highest intensity due to low concentration of defects which act as trap states that offer energy level in between HOMO and LUMO [5]. 

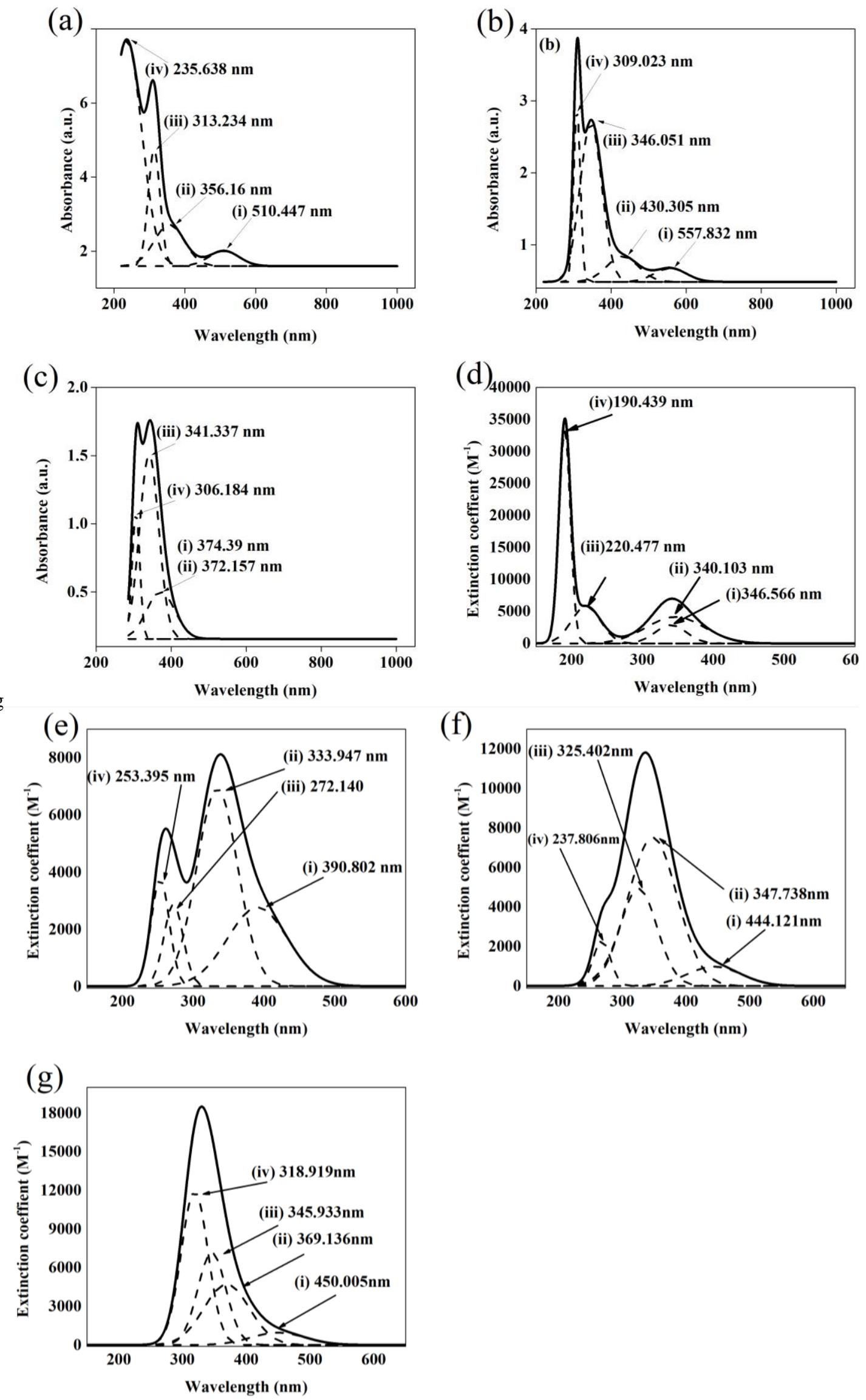

Figure 3: The excitonic peaks of $\mathrm{ZnS}$ thin films fabricated at a vacuum pressure of (a) $10^{-5}$ Torr, (b) $10^{-6} \mathrm{Torr}$, (c) $10^{-7}$ Torr. The simulated excitonic peaks of modelled clusters of $(\mathrm{d})(\mathrm{ZnS})_{3}$, (e) $(\mathrm{ZnS})_{6}$, (f) $(\mathrm{ZnS})_{9}$, and $(\mathrm{g})(\mathrm{ZnS})_{12}$ which all have been fitted to four Gaussian peaks. 

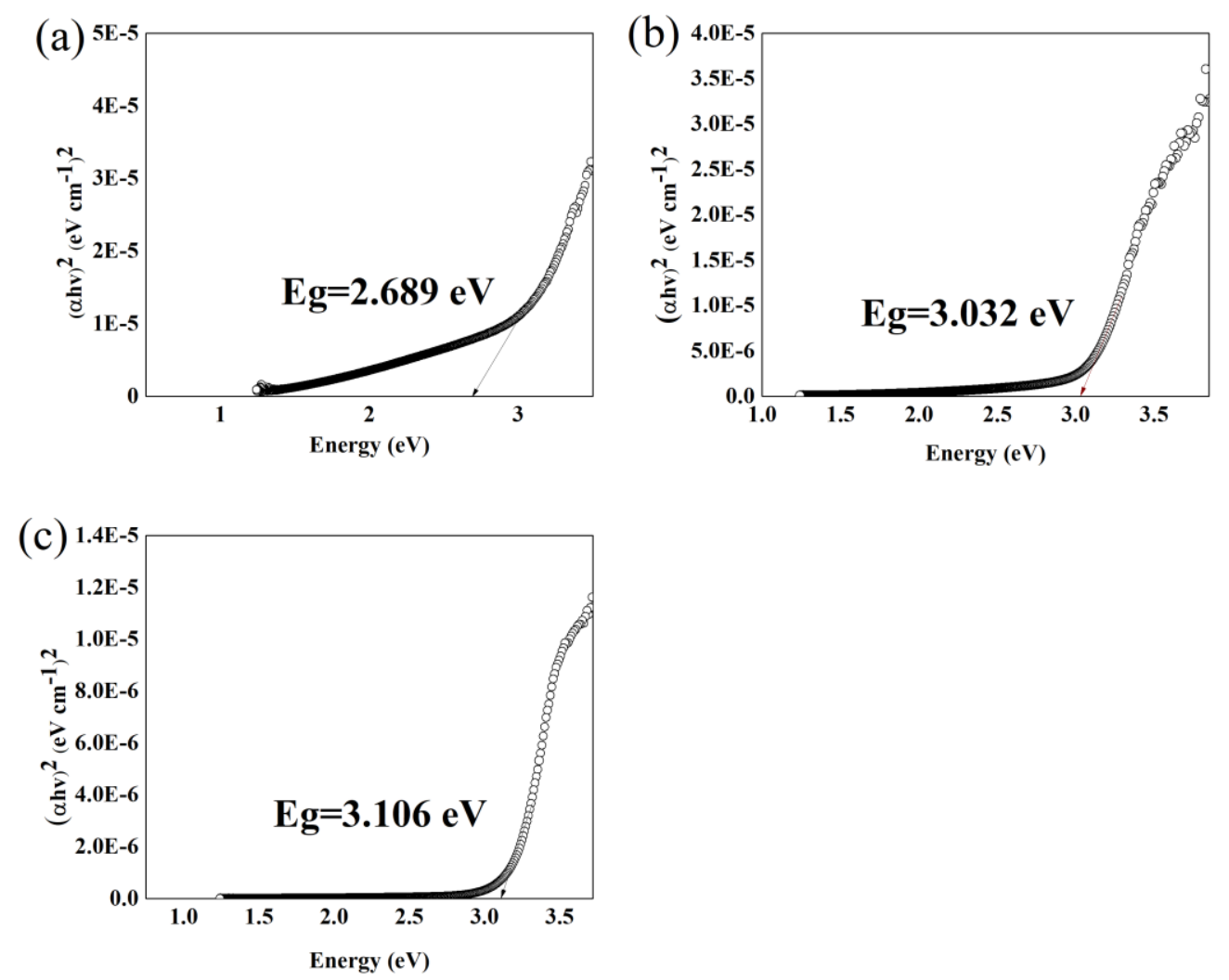

Figure 4: Tauc's plots of ZnS thin films fabricated at a vacuum pressure of (a) $10^{-5}$ Torr, (b) $10^{-6}$ Torr, and (c) $10^{-7}$ Torr.
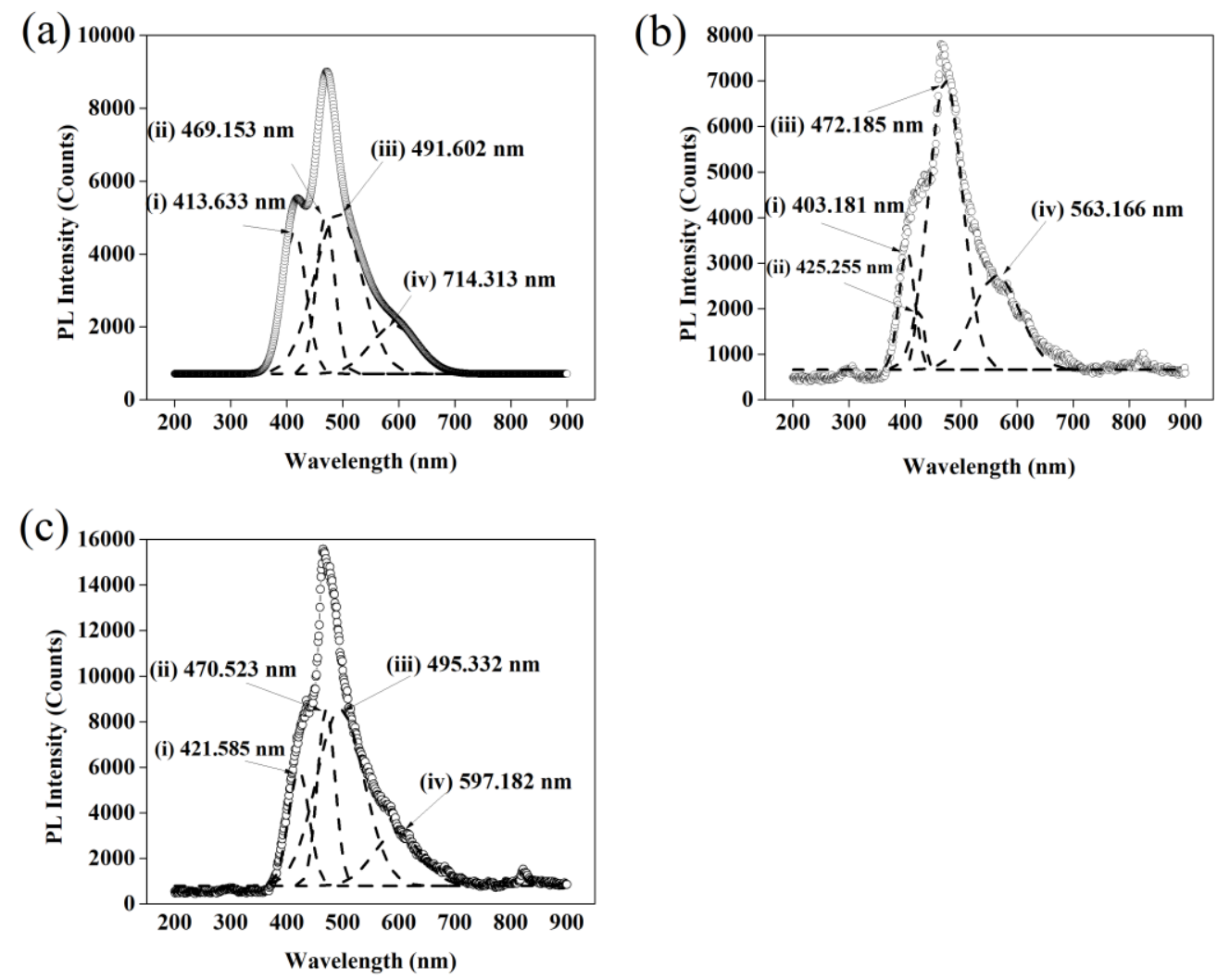

Figure 5: Photoluminescence peaks of $\mathrm{ZnS}$ thin films fabricated at a vacuum pressure of (a) $10^{-5}$ Torr, (b) $10^{-6}$ Torr, and (c) $10^{-7}$ Torr which have been fitted to four Gaussian peaks. 


\section{CONCLUSION}

Optical properties and crystal structures of $\mathrm{ZnS}$ thin films fabricated using thermal evaporators at different vacuum pressures have been studied. The $\mathrm{ZnS}$ thin film fabricated at a vacuum pressure of $10^{-7}$ Torr is in good agreement with realistic clusters of $(\mathrm{ZnS})_{3}$ and $(\mathrm{ZnS})_{6}$. $\mathrm{ZnS}$ thin film fabricated under vacuum pressure of $10^{-7}$ Torr is having the highest PL intensity, due to the low percentage of trap states originated by defects. Furthermore, the decrement of vacuum pressure during the thermal evaporation process would produce $\mathrm{ZnS}$ thin films with altered band gap i.e., the lower the pressure, the wider the bandgap; which is speculated due to the decrement of the size of the basic building block of the $\mathrm{ZnS}$ particles in each thin films.

\section{ACKNOWLEDGEMENT}

This work is funded by the Research \& Innovation Department of Universiti Malaysia Pahang, Universiti Teknologi MARA, and the International Islamic University of Malaysia through Sustainable Research Collaboration Grant (RDU 200711).

\section{REFERENCES}

[1] X. Fang et al., "ZnS nanostructures: From synthesis to applications," Prog. Mater. Sci., vol. 56, no. 2, pp. 175287, 2011, doi: https://doi.org/10.1016/j.pmatsci.2010.10.001.

[2] L. Abdallah and T. El-Shennawy, "Reducing Carbon Dioxide Emissions from Electricity Sector Using Smart Electric Grid Applications," J. Eng., vol. 2013, p. 845051, 2013, doi: 10.1155/2013/845051.

[3] L. I. Berger, Semiconductor materials. CRC press, 1996.

[4] N. Soltani et al., "Visible Light-Induced Degradation of Methylene Blue in the Presence of Photocatalytic ZnS and CdS Nanoparticles," Int. J. Mol. Sci., vol. 13, no. 12, pp. 12242-12258, Sep. 2012, doi: 10.3390/ijms131012242.

[5] F. F. A. Azmi, S. K. Muzakir, M. F. Z. Kadir, and S. B. Aziz, "On the structural-optical correlation of ZnO nanospheres synthesized using thermal evaporation technique," Mol. Cryst. Liq. Cryst., vol. 693, no. 1, pp. 6675, Nov. 2019, doi: 10.1080/15421406.2020.1723912. 